Sylvain Delcomminette, Pieter D’Hoine, MarcAntoine Gavray (éd.), Ancient Readings of Plato's Phaedo

\title{
Giovanni Casertano
}

\author{
(2) OpenEdition \\ Journals \\ Edizione digitale \\ URL: https://journals.openedition.org/philosant/689 \\ DOI: $10.4000 /$ philosant.689 \\ ISSN: 2648-2789 \\ Editore \\ Éditions Vrin
}

\section{Edizione cartacea}

Data di pubblicazione: 1 novembre 2016

Paginazione: 213-218

ISBN: 978-2-7574-1472-9

ISSN: 1634-4561

\section{Notizia bibliografica digitale}

Giovanni Casertano, «Sylvain Delcomminette, Pieter D'Hoine, Marc-Antoine Gavray (éd.), Ancient Readings of Plato's Phaedo », Philosophie antique [En ligne], 16 | 2016, mis en ligne le 01 novembre 2018, consulté le 02 décembre 2022. URL : http://journals.openedition.org/philosant/689; DOI : https://doi.org/10.4000/philosant.689

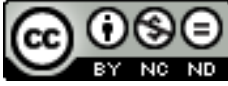

Creative Commons - Attribution - Pas d'Utilisation Commerciale - Pas de Modification 4.0 International - CC BY-NC-ND 4.0

https://creativecommons.org/licenses/by-nc-nd/4.0/ 
leur nouveauté et leur excellence. Ce recueil illustre pleinement la vitalité des études hippocratiques et le succès de l'entreprise lancée par Jacques Jouanna en 1972. La $15^{\mathrm{e}}$ édition a eu lieu à Manchester en octobre 2015 sur le thème du commentaire.

Antoine Pietrobelli

Université de Reims Champagne Ardenne

Sylvain Delcomminette, Pieter D'Hoine, Marc-Antoine Gavray (éd.), Ancient Readings of Plato's Phaedo, Leiden-Boston, Brill, 2015 (Philosophia antiqua, 140), 364 p., ISBN : 978-90-04-28217-9.

Questo libro, come chiariscono i curatori, è il risultato di un progetto scientifico sullo studio dei commentari neoplatonici del Fedone, già realizzatosi in un convegno del 1970 diretto da Westerink, e poi ampliatosi in un convegno sulla storia delle interpretazioni del dialogo nell'antichità realizzatosi nel 2012 a Bruxelles: non più solo platonici, ma anche aristotelici, stoici, scettici.

Dopo un' «Introduzione», redatta dai tre editori, i saggi raccolti sono 13, a cura di Sylvain Delcomminette («Aristote et le Phédon»), Han Baltussen ( «Strato of Lampsacus as a reader of Plato's Phaedo: his critique of the soul's immortality»), Francesca Alesse («Le Phédon dans le stoïcisme hellénistique et post-hellénistique»), Lorenzo Corti («Sextus, the number two and the Phaedo»), Geert Roskam («Plutarch's reception of Plato's Phaedo»), Harold Tarrant («The Phaedo in Numenian allegorical interpretation»), Riccardo Chiaradonna («Plotin lecteur du Phédon: l'âme et la vie en IV 7 [2], 2»), Pieter d'Hoine ( «Syrianus and the Phaedo»), Alain Lernould («Damascius, Olympiodore et Proclus sur les attributs "divin" ( $\theta \varepsilon \hat{\varepsilon}$ ov) et "intelligible" (vontóv) en Phédon 80a10-b1 dans l'argument dit "de la similitude" »), Sebastian Geertz («From "immortal" to "imperishable": Damascius on the final argument in Plato's Phaedo»), Franco Trabattoni ( «La théorie de l'âme-harmonie chez les commentateurs anciens»), Bram Demulder e Gerd Van Riel ( «"Nombreux sont les porteurs de thyrse, mais rares les Bacchants": Olympiodore et Damascius sur le Phédon»), Marc-Antoine Gavray («Au terme d'une tradition: Simplicius, lecteur du Phédon»). Il volume comprende infine una bibliografia, e tre indici, locorum, nominum, rerum.

Nell'Introduzione i curatori individuano tre diverse modalità di approccio al dialogo platonico: un atteggiamento critico, che è proprio di Aristotele, di Stratone (terzo scoliarca del Liceo), e poi di Stoici e Scettici; un approccio di tipo esegetico, che si ritrova in particolare nei commentari del platonismo di età imperiale, allorché il commentario era divenuto il genere filosofico per eccellenza; un uso del dialogo nel contesto di costruzione del pensiero proprio dell'interprete, in certo senso una vera e propria «appropriazione» del dialogo. Quest'ultimo atteggiamento ermeneutico a sua volta viene distinto in tre tipi: un'appropriazione al livello storico (il dialogo inteso come documento storico sulla vita di Socrate, come accade con gli Stoici; o come apertura alla comprensione delle dottrine orfiche e pitagoriche, come accade con Numenio); un'appropriazione al livello letterario o retorico, legato comunque al precedente, come accade con il medioplatonismo, e in particolare con Plutarco; un'appropriazione a livello filosofico, come accade con i tardi commentatori neoplatonici, ma anche con alcuni interpreti critici del dialogo (Aristotele, Stoici). Il criterio proposto dai curatori è molto interessante, e credo che abbia valore non solo riguardo al caso particolare del Fedone e delle sue letture nel corso dei secoli, ma anche più in generale come criterio di interpretazione storiografica. Ma qui non lo seguirò, bensì darò una rapidissima scorsa ai vari saggi nell'ordine in cui appaiono nel volume. 
Molto lucido ed esauriente l'articolo di Delcomminette, che mette in luce come l'interesse di Aristotele non si sia focalizzato sul tema dell'anima: ci sono poche citazioni del Fedone, delle quali una sola nel De anima, mentre le altre rinviano all'autobiografia di Socrate e alla cosmologia del mito finale. Fa eccezione naturalmente l'Eudemo, nel quale sono numerosi i temi ripresi dal Fedone, e fra questi in particolare la critica della tesi dell'anima-armonia, l'unico tema che rimane nel De anima. Ragion per cui, per cercare l'influenza del Fedone e di Platone in generale nell'opera aristotelica, Delcomminette esamina una serie di passi in cui né il dialogo né Platone sono citati esplicitamente e nota come l'influenza del dialogo sulla filosofia aristotelica sia profonda, ma «spostata», per così dire, dal campo della psicologia a quello della scienza della natura. Esempi di come l'appropriazione critica aristotelica si rifaccia al Fedone si può trovare nella sua recezione dei passi in 60b-c e 70a-72e, dove c'è la distinzione fondamentale tra contrari in se stessi e cose contrarie che li accolgono; e nel passo in 103c-105e, dove Aristotele vede la prefigurazione della sua distinzione tra predicazione accidentale e predicazione essenziale.

Decisamente critica nei confronti del Fedone, e in particolare della dimostrazione dell'immortalità dell'anima, è la posizione dell'aristotelico (ma non ortodosso: critica fra l'altro la concezione aristotelica del tempo) Stratone di Lampsaco. Baltussen mostra innanzitutto la complessità delle operazioni di destratificazione che bisogna mettere in campo per poter delineare le linee della sua critica a Platone: non possedendo nessuno scritto di Stratone, noi leggiamo le sue obiezioni al dialogo nel commentario di sette secoli dopo attribuito al neoplatonico Damascio, il quale, a sua volta, vuole confutare Stratone servendosi anche di un retroterra di argomentazioni e di discussioni interne al platonismo, attraverso Plutarco, Polibio, Proclo. Si tratta cioè, suggerisce con un'efficace immagine Baltussen, di una matrioska russa; un insieme di scatole cinesi, diremmo noi, delle quali non è facile venire a capo. Una cosa è certa: l'insieme delle critiche a Stratone mostra se non altro quanto fossero tenute in considerazione da coloro che volevano difendere a tutti i costi la «verità» platonica (anche se questa verità platonica era la propria più che quella di Platone). Come che sia, le argomentazioni polemiche di Stratone al dialogo platonico riguardano principalmente il tema dell'immortalità dell'anima, a partire dal cosiddetto argomento degli opposti: fondamentalmente, le obiezioni di Stratone vogliono mostrare che le argomentazioni platoniche dimostrano solo che l'anima non può ammettere la morte, ma non che l'anima sopravvive al corpo; che l'anima non muoia non significa che non cessi di esistere, ma solo che non può esistere in uno stato morto. Baltussen sottolinea efficacemente il retroterra aristotelico delle obiezioni di Stratone, specialmente per quanto riguarda quella che chiama la sua teoria «fisicalista» della psicologia e dell'attività cognitiva: corpo e anima, ambedue di natura fisica, interagiscono continuamente, per cui non v'è opposizione tra intelletto e sensi. Il legame con gli atomisti è chiaro; ma non capisco perché Baltussen definisce il fisicalismo di Stratone «più sottile» di quello degli atomisti: anche con Democrito (e sia pure deducendolo dalle numerose pagine che gli dedica, ma a volte con aperta ammirazione, il critico Aristotele) siamo di fronte ad una teoria della conoscenza e dell'anima tutt'altro che grossolana.

Decisamente polemici nei confronti di Platone, anche se ammiratori di Socrate (sorvolando sul fatto che la figura di Socrate è un'invenzione di Platone), sono gli Stoici. Francesca Alesse affronta la questione della presenza dei dialoghi platonici, e in particolar modo del Fedone, nei frammenti stoici; questione complicata, perché in effetti nei testi stoici non vi sono citazioni esplicite e dirette. Ma la Alesse, in Aristone, Crisippo, Seneca ed Epitteto, ci fa vedere come, nonostante alcune tesi centrali del dialogo (la separazione 
dell'anima dal corpo, la dottrina della reminiscenza) siano decisamente contrarie alla filosofia stoica, metafore, termini tecnici e argomentazioni si possono far risalire al dialogo. Così avviene, per esempio, per l'unicità della virtù in Aristone; per l'uso, in Crisippo, di versi omerici (citati in Phaed. 94d8-9), citati per giungere ad una posizione contraria a quella di Platone. Ma la vicinanza, potremmo dire di «atmosfera», al dialogo platonico si fa più stretta nello stoicismo romano, con Seneca ed Epitteto, specialmente per quanto riguarda le immagini relative al rapporto tra anima e corpo e la riflessione sulla morte. Problematica rimane invece, per Alesse, la notizia che in ambiente stoico il Fedone non è stato giudicato autentico: non c’è alcuna ipotesi plausibile per giustificare il perché Panezio abbia ritenuto inautentico il dialogo (e non ne possiamo nemmeno essere certi).

Lorenzo Corti mostra come una marginale ma interessante menzione del dialogo si trova in Sesto, in particolare su come il numero due si può formare dall'unione di unità (Phaed.96e-97b). Sesto usa alcune questioni del dialogo contro la nozione dogmatica del numero due: il numero due non esiste, così come non esiste l'uno o l'unità; l'introduzione delle idee, e quindi dell'idea del due, dimostra per Sesto che Socrate ignora la questione di come nasca il due. Incidentalmente, ma giustamente, poi Corti sostiene, sulla scia di Burkert, che la dottrina dei due principi e del sistema che ne deriva non è dottrina pitagorica, ma dell'antica Accademia.

Ben articolato e convincente è il saggio di Roskam sulla recezione plutarchea del Fedone, che ha esercitato una notevole influenza sul pensiero e sulla scrittura di Plutarco. Recezione comunque complessa: in alcuni casi si tratta di citazioni letterali e di chiari riferimenti, in altri di vaghe allusioni, con molti elementi ripresi da altri autori e altre fonti. Viceversa, chiari riferimenti al Fedone appaiono dove non ce li aspetterebbe: Plutarco in effetti usa il dialogo in contesti molto differenti. Per queste ragioni risulta difficile definire con precisione l'influenza del Fedone in particolari passaggi delle opere di Plutarco (l'A. comunque fornisce anche un elenco dei passi e delle corrispondenze). La grande importanza del Fedone, ma anche di altri dialoghi, per Plutarco, è dimostrata non solo dal fatto che viene usato come fonte dottrinale, ma anche come modello letterario e narratologico. Non è da sottovalutare, infine, il fatto che le interpretazioni plutarchee ebbero una grande influenza su altre scuole filosofiche e in particolare sul tardo platonismo. Il saggio si chiude con una intelligente, simpatica ed autoironica notazione dell'A.: il Fedone fu soltanto uno degli ingredienti di una salsa estremamente sofisticata, preparata da Plutarco, per cui sarebbe molto meglio ignorare il processo di composizione della salsa e gustarla direttamente. Che è come a dire che è meglio ignorare questo saggio e procedere alla lettura diretta di Platone e di Plutarco.

Con Numenio, nel II d.C., iniziano le interpretazioni allegoriche del dialogo, e degli antichi in genere, a cominciare da Omero. Il saggio di Tarrant ricorda che Numenio non è conosciuto come autore di commentari platonici, ma la sua lettura di Platone è significativa di quella tendenza propria di certi ambienti culturali del suo secolo a considerare Platone parte integrante della tradizione di saggezza pitagorica. Un pitagorico, dunque, ma che può essere incluso tra i platonici in contesti di interpretazioni di Platone: Platone è d'accordo con Pitagora.

Il ruolo di Plotino come lettore del Fedone è esaminato da Riccardo Chiaradonna. Il dialogo è tra i più presenti nelle Enneadi, ma l'A. ne commenta un solo passo, quello in IV $7[2], 2$, nel trattato appunto sull'incorporeità dell'anima e la sua immortalità. Plotino combina le prove del Fedone con l'argomento del Fedro sull'anima e il movimento, e la prova gioca un ruolo chiave in questa sezione. L'anima è una cosa tale che la vita le è presente «in se stessa» e quindi non può perire; la contestazione del peripatetico 
Stratone, sull'illeceità del passaggio dall'immortalità all'indistruttibilità viene respinta con un «completamento» dell'argomento platonico: l'anima non è solo essenzialmente vivente, ma essenzialmente esistente e di conseguenza è non solo immortale ma anche indistruttibile.

Passando quindi ai commentatori neoplatonici del v secolo, un posto importante nella storia delle interpretazioni è quello dell'ateniese Siriano, oggetto dello studio di Pieter d'Hoine. Non ci sono prove conclusive che Siriano abbia scritto un intero commentario al Fedone, ma non può essere escluso che sia circolato sotto il nome di Siriano un commentario al dialogo. La sua interpretazione è quindi ricostruita sulla base dei commentari di Damascio e Olimpiodoro. Tutti i loro riferimenti riguardano l'argomento dei contrari, per cui possiamo supporre che si possa attribuire a Siriano una monografia sull'argomento. In genere, Siriano è polemico nei confronti di Aristotele; a proposito per esempio dell'argomento della reminiscenza, egli la interpreta nel senso che le forme nell'anima, superiori ai particolari sensibili, costituiscono un'alternativa alla visione aristotelica della formazione dei concetti per via di astrazione.

La riflessione di Damascio, Olimpiodoro e Proclo sugli attributi di «divino» e «intelligibile» nell'argomento «della similitudine» è l'oggetto dello studio di Lernould. I neoplatonici vedono nella seconda serie dei sei attributi (Phaed. 80a10b7) le determinazioni delle forme che «divengono», cioè quelle insite in una materia, distinguendo così le Forme che sono da quelle che divengono: nel far così, Damascio sostituisce nel testo platonico «corporeo» a «umano». Ma non è l'unica forzatura del testo platonico: sia Damascio che Olimpiodoro danno un senso attivo a vontóv: quale ragione filosofica può giustificare questa violenza grammaticale? L'A. individua in Plotino e in Proclo l'origine di questa interpretazione: Plotino insisteva sull'idea che le Forme nell'intelletto divino non sono soltanto passivamente «pensate», ma sono esse stesse pensieri in atto. Ed Olimpiodoro ci fornisce anche l'opinione di Proclo sulla questione di sapere di quali forme parla Platone, quelle che sono nel demiurgo o quelle nell'anima umana: Proclo pensa che si tratti di due tipi diversi, perché le forme intellettive sono i modelli di quelle che sono nell' anima. Alla base di Olimpiodoro e di Damascio c'è dunque Proclo, con il suo sostituire il divino alle forme e fare dell'argomento della similitudine con le forme un argomento della similitudine col divino. Il tutto per dimostrare come, a partire dal Fedone, è possibile elaborare una completa teologia platonica: il passo del dialogo è depositario di una parte integrale di una dottrina teologica completa, e il suo contenuto filosofico è rapportato, a lato di altri dialoghi, all'iniziazione e ai misteri divini.

Sebastian Geertz esamina le critiche di Damascio a Stratone, e in particolare la negazione che questi fa dell'equivalenza tra immortale e indistruttibile. In effetti i neoplatonici furono colpiti dalle critiche di Stratone; c'era la pratica di includere la discussione delle obiezioni antiplatoniche nei commentari: quello di Damascio conserva alcune delle contro-obiezioni di Proclo a Stratone.

Proclo e Damascio distinguono tra due livelli di vita, 1) la vita che possiede l'anima che porta vita, 2) la vita che è provocata dall'anima. Se si assume che la vita che possiede l'anima è differente da quella che porta nei corpi, allora essa non può soffrire lo stesso tipo di morte che soffrono gli esseri animati, e per lei morire significa semplicemente separarsi dal corpo.

Le interpretazioni neoplatoniche di Filopono e di Damascio della confutazione socratica della tesi dell'anima-armonia sono oggetto del saggio di Franco Trabattoni. L'A. vuole dimostrare che le spiegazioni dei due neoplatonici si basano su un'errata lettura del testo platonico, e in particolare delle linee 93a-94a, offrendo anche una propria ricostruzione del passo. Si tratta del passo in cui Socrate rifiuta la teoria dell'anima- 
armonia basandosi sull'analogia tra armonia e anima. Per Damascio è vero che l'armonia ha dei gradi, ma questo significa che non è una sostanza, ma una qualità. Per Filopono, l'argomento principale è che l'armonia ammette il più e il meno, l'anima no; l'anima ammette la virtù e il vizio, ma l'armonia non può ammettere l'armonia e la mancanza di armonia. Filopono e Damascio sono poi in disaccordo sulla questione se Socrate abbia affermato o abbia negato la possibilità che l'armonia abbia dei gradi. Filopono pensa all'affermazione; Damascio alla negazione. I problemi si risolvono, secondo Trabattoni, se si interpreta il passo platonico nel suo senso: esso non nega né afferma che l'armonia abbia dei gradi, ma vuole stabilire l'equivalenza dei gradi di armonizzazione e dei gradi di armonia.

In un articolo molto interessante Demulder e Van Riel si pongono il compito di paragonare i commentari di Olimpiodoro e Damascio, mettendoli in relazione all'ambiente culturale alessandrino e a quello ateniese, nonché alle rispettive posizioni di fronte alla cultura cristiana. Comune ai due neoplatonici era la deplorazione della perdita di cultura della propria epoca, più che una aperta reazione al cristianesimo. Comune era anche il modello scelto per i propri commentari: Proclo. Su queste basi però i due Autori fanno anche vedere le differenze che ci sono tra i due neoplatonici, che a volte sono di non poca importanza. Nella scuola di Alessandria, il commentario di Olimpiodoro si presentava come un'opera pedagogica per guidare gli allievi interessati, ma non specializzati negli studi filosofici, mentre ad Atene Damascio si rivolgeva ad un pubblico iniziato. Interessanti sono le differenze delle interpretazioni dei due neoplatonici, in particolare di due passi: 61c-62b, il passo sulla frourà e sul divieto della fuga, e la discussione delle virtù in $68 \mathrm{c}-69$ e. Così, a proposito poi della formula orfica («molti sono i portatori di tirso, pochi i bacchi») utilizzata da Socrate, Damascio spiega che il tirso era una forma fallace che rinviava a virtù fallaci. E poi continua esponendo $i$ riti d'iniziazione e il carattere mistico della vera filosofia: la filosofia è una via verso l'alto che gli allievi devono seguire. Altra storia in Olimpiodoro: coloro che si occupano di politica sono i portatori di tirso, e coloro che sono nella fase purificatrice sono i baccanti, solo che non si rigetta il tirso divenendo baccanti: le virtù politiche devono essere purificate, e il vero filosofo deve combinare le due vie. Contrariamente a Damascio, egli mostra simpatia per la figura del politico: mentre per Damascio non può essere definito makarios chi è pervenuto alle virtù politiche, Olimpiodoro sostiene che può esserlo anche chi ha raggiunto il livello delle virtù politiche.

E alla fine di questo lungo viaggio, durato quasi mille anni, incontriamo Simplicio: la sua lettura del Fedone è l'oggetto del saggio di Gavray. Discepolo di Damascio, Simplicio, della scuola di Atene, non ha scritto commentari al dialogo, ma comunque l'A. si propone di rintracciare nella sua opera quanto può essere individuato come suo apporto personale all'interpretazione del Fedone, nonché la sua distanza da Damascio, nonostante i loro metodi e obiettivi fossero analoghi: ciascun dialogo è un esercizio mirante a sviluppare un certo tipo di virtù, e il Fedone deve arricchire le virtù catartiche del lettore, aiutandolo a raggiungere la purificazione, cioè la separazione dell'anima dal corpo. Rispondendo ad un'obiezione sollevata da Alessandro di Afrodisia, secondo la quale l'argomento dei contrari per l'immortalità dell'anima è puramente verbale, Simplicio distingue la diversa portata delle prove sull'immortalità, una valida perché procede a partire dall'essenza dell'anima e della sua natura automotrice, l'altra verbale perché procede da premesse generali che non sono specificamente associate all'anima. Il suo intento generale, di integrare l'esegesi platonica del Fedone alla sua lettura di Aristotele, risulta evidente anche dalla sua interpretazione del mito finale del dialogo. A differenza di Alessandro, però, che dà ragione ad Aristotele contro Platone, Simplicio cerca di conciliare i due. 
Concludendo, siamo di fronte a un libro molto interessante, che dimostra almeno due cose. In primo luogo, la grande importanza che il Fedone ha avuto nella storia delle interpretazioni platoniche, fin dall'antichità, mostrando con ciò di essere, a tutti gli effetti, un vero classico della letteratura filosofica di tutti i tempi.

La seconda cosa che si nota è la conferma di un fatto ben noto a chi si interesssa di storia della storiografia: tutti gli interpreti hanno usato il Fedone come uno strumento ritenuto importante per polemizzare con altre scuole di pensiero, e si sono quindi appropriati del testo piegandolo ai propri orientamenti filosofici. In altri termini, il testo platonico è stato al centro di una grande varietà di approcci, ognuno dei quali, anche nel criticarlo, lo riteneva importante per l'affermazione della propria prospettiva.

Giovanni Casertano Università di Napoli «Federico II»

Mauro Bonazzi, Il platonismo, Turin, Giulio Einaudi editore, 2015 (Piccola Biblioteca Einaudi, 56), 239 p., ISBN : 978-88-06-21689-4.

Le terme platonisme est ambigu : il peut désigner soit le contenu de la philosophie de Platon, soit l'histoire de sa réception et de ses interprétations. C'est en suivant les méandres de cette seconde acception que Mauro Bonazzi (MB) propose une étude du platonisme dans un parcours allant de Speusippe jusqu'aux derniers diadoques de l'école d'Alexandrie. L'ouvrage, qui entre rapidement dans son objet, comporte quatre chapitres bien équilibrés : 1. «L'Académie antique » (p. 3-37); 2. «Platonisme et scepticisme ? L'Académie à l'époque hellénistique » (p. 38-72); 3. «Vers le système : le platonisme de la première époque impériale » (p.73-109); 4. « Le triomphe du système : le néoplatonisme » (p. 110-160). Suivent deux appendices : (1) « Le platonisme et la politique $\gg($ p. 163-179) qui a pour but de contrebalancer la vision d'un platonisme uniquement contemplatif et de réévaluer non seulement le rôle que les différents scholarques jouèrent sur la scène publique, mais encore la teneur du message politique des platoniciens anciens; (2) «Le platonisme et le christianisme » (p. 180-194) qui propose un aperçu très bien documenté de l'évolution des rapports entre christianisme et néoplatonisme de Porphyre jusqu’à saint Augustin ; une bibliographie complète et actualisée ; un index nominum.

Sous l'apparente linéarité de l'exposition, l'ouvrage relève un défi de taille : ôter les lunettes dogmatiques qui font voir une essence pérenne du platonisme et déduisent par après ce qui n'en constitue que des dérives accidentelles. En d'autres termes, il s'agit de montrer qu'il n'y a pas d'option préférentielle sur ce que devrait être le platonisme. Dès la première page, l'auteur souligne que mettre la pensée de Platon en système est le fruit d'une reconstruction qui prend forme à l'époque impériale. Le platonisme ne peut se dire qu'au pluriel. Le livre de $\mathrm{MB}$ examine la façon dont, sur près d'un millénaire, les exégètes ont tenté de reconstruire ce qui était censé être la doctrine véritable de Platon en indiquant que le caractère protéiforme des figures qu'il a prises au cours des siècles découle de difficultés internes aux dialogues eux-mêmes. La lecture intégrative de MB fait surgir quelques paradoxes, comme celui consistant à défendre que, tout autant que le dogmatisme impérial, le scepticisme académicien est à inclure dans l'héritage platonicien.

L'auteur procède méthodiquement en rappelant que dans l'Ancienne Académie ne régnait aucune orthodoxie, mais que s'y exprimait une pluralité de courants interprétatifs. Tous ont pour point de départ la philosophie de Platon qui non seulement a ouvert la voie à des directions multiples au sein de la tradition platonicienne, mais aussi a fait naître des philosophies dissidentes, l'aristotélisme, puis le stoïcisme. C'est à cette époque 